\title{
THE ROLE OF DIAGNOSTIC OESOPHAGO-GASTRODUODENOSCOPY (OGD) AND COLONOSCOPY IN CANCER OF UNKNOWN PRIMARY
}

\author{
Muhammad Ismail, Rao Saad Ali Khan, Farrukh Saeed, Muhammed Aasim Yusuf* \\ Pak Emirates Military Hospital/National University of Medical Sciences (NUMS) Rawalpindi Pakistan, *Shaukat Khanum Memorial Cancer Hospital \& Research \\ Centre, Lahore Pakistan
}

\begin{abstract}
Objective: To study the role of gastrointestinal procedures, namely oesophago-gastroduodenoscopy (OGD) and colonoscopy, in helping to establish a definitive primary tumour site in cancer of unknown primary.

Study Design: Prospective observational study.

Place and Duration of Study: Shaukat Khanum Memorial Cancer Hospital \& Research Centre, Lahore Pakistan, from Jan 2018 to Jan 2019.

Methodology: A total of 115 patients included in the study were those, who underwent OGD and a colonoscopy for the diagnosis of a cancer of unknown primary. Data collected included demographics, baseline clinical characteristics, definitive diagnosis, tissue diagnosis and immune-histochemical stains. Primary outcome was the attainment of a definitive diagnosis via OGD and/or colonoscopy.

Results: A total of 115 patients underwent a diagnostic gastrointestinal procedure. Of these 70 (61\%) were males. Mean age was $63 \pm 12.6$ years (range 22-88 years). Abdominal pain comprised the most common presenting complaint, found in 61 (53\%). The most common tissue diagnosis of the metastatic sites was adenocarcinoma $81(70.45 \%)$. Tumour markers including carcinoembryonic antigen, alpha-fetoprotein and carbohydrate antigen 19-9 were checked in 90 (78.2\%), 46 (40\%) and 69 (60\%) patients respectively. No patient reached a definitive diagnosis by means of OGD and/or colonoscopy.

Conclusion: OGD and colonoscopy when done collectively as diagnostic procedures to look for a primary tumour, have no value in the evaluation of patients with cancer of unknown primary.
\end{abstract}

Keywords: Cancer of unknown primary (CUP), Colonoscopy, Oesophago-gastroduodenoscopy (OGD).

How to Cite This Article: Ismail M, Khan RSA, Saeed F, Yusuf MA. The Role of Diagnostic Oesophago-Gastroduodenoscopy (OGD) and Colonoscopy in Cancer of Unknown Primary. Pak Armed Forces Med J 2021; 71(6): 2166-2169. Doi: https://doi.org/10.51253/pafmj.v71i6.4090

This is an Open Access article distributed under the terms of the Creative Commons Attribution License (https://creativecommons.org/licenses/by-nc/4.0/), which permits unrestricted use, distribution, and reproduction in any medium, provided the original work is properly cited.

\section{INTRODUCTION}

Carcinoma of unknown primary site (CUP) is the $10^{\text {th }}$ most common presentation of cancers seen globally, accounting for $3-5 \%$ of all malignancies. ${ }^{1}$ It is defined as a heterogeneous group of cancers in which one or more metastatic sites are found but the primary site of origin cannot be identified. ${ }^{2-4}$ It is broadly classified into adenocarcinoma, squamous cell carcinoma, poorly differentiated malignant neoplasm, neuroendocrine carcinoma and lymphoma. ${ }^{5}$ The most common of these is adenocarcinoma and it often has a poor prognosis. ${ }^{2,6}$

The detection of the primary site of a tumour requires a thorough history and clinical examination, followed by investigation, usually with radiological techniques, often supplemented by endoscopic procedures. Biopsy with additional immunohisto-chemistry studies and tumour markers is usually performed. ${ }^{6-9}$ Finding the primary site of a tumour has both therapeutic and prognostic implications ${ }^{2}$ and so an approp-

Correspondence: Dr Muhammad Ismail, Fellow Gastroenterology, Pak Emirates Military Hospital, Rawalpindi Pakistan

Received: 09 Apr 2020; revision received: 26 Jun 2020; accepted: 02 Jul 2020 riate diagnostic workup is an important aspect of patient care. ${ }^{10}$

Endoscopy has a low specificity and sensitivity in diagnosing primary tumour in patients with $\mathrm{CUP}^{6}$ and its use should therefore be guided by appropriate signs and symptoms. ${ }^{10}$ Limited studies are available to date regarding the yield of upper and lower GI endoscopic procedures in reaching a definitive diagnosis in patients with CUP. We therefore aimed to ascertain the role of endoscopic procedures, namely OGD and colonoscopy, in establishing a definitive diagnosis in CUP.

\section{METHODOLOGY}

This was a prospective observational study evaluating patients seen at Shaukat Khanum Memorial Hospital and Research Centre Lahore, from January 2018 and January 2019. Approval from the hospital's institutional review board (IRB approval no. 02-01-1801) was obtained prior to commencing data collection. We recorded patient demographics including age, gender, clinical presentation, imaging studies, immunohistochemical staining, and tissue diagnosis of malignancy. We also recorded the definitive diagnosis, if 
achieved. We included all the patients from January 2018 to January 2019 through convenient sampling technique.

Inclusion Criteria: Patients who had a tissue diagnosis of a metastatic malignancy, without a definitive primary site were included.

Exclusion Criteria: Patients, who were less than 18 years of age, were excluded.

Based on these criteria, a total of 115 patients fulfilled the inclusion criteria. These patients had a clinical history, imaging studies, or immunohistochemistry suggestive of a possible gastrointestinal (GI) primary site.

All the patients included in the study underwent both an OGD and a colonoscopy. The procedures were done by or under the direct supervision of a consultant gastroenterologist, under conscious sedation, with monitoring of pulse, blood pressure, oxygen saturation at five-minute intervals throughout the procedure. The various endoscopic findings were recorded and any lesion/area considered to be suspicious was subjected to biopsy. The primary outcome of our study was the attainment of a definitive diagnosis in patients with CUP by the use of OGD and colonoscopy.

Detailed demographics, history and physical examination were carried for all patients prior to their endoscopic procedures. Qualitative and quantitative variables, including age, gender, symptoms and imaging characteristics were recorded. Mean, median, frequencies and standard deviations values were calculated where applicable. The proportion of tissue diagnoses obtained by OGD and colonoscopy was also recorded as a quantitative variable. All the data were collected and analyzed on the Statistical Package for the Social Sciences (SPSS) version 20 (International Business Machines, IBM corporation).

\section{RESULTS}

A total of 115 patients were included in the study, each of whom had an OGD and a colonoscopy. Thus a total of 230 procedures were performed. Out of 115 patients, $70(61 \%)$ were males. The mean age at presentation was $63 \pm 12.6$ years (range, 22-88 yrs) and 87/115 patients had gastrointestinal symptoms. Abdominal pain comprised the most common presenting complaint, occurring in 61 (53\%) of patients, while other GI symptoms included weight loss, altered bowel habit, dysphagia, anorexia, nausea and vomiting as shown in Table-I. While 28 patients had no GI symptoms, but had OGD and colonoscopy in view of either raised tumour markers or immunohistochemical (IHC) stains and/or imaging features suggestive of a GI tract primary. The various imaging modalities that were used in our patients included computerized tomography (CT), magnetic resonance imaging (MRI), ultrasound scan and positron emission tomography (PET) scan. Among these, CT was the most commonly employed imaging modality, being used in 107 (93\%) of patients. The most common site of metastases was liver 40 $(34.8 \%)$, followed by metastases in multiple sites in $19(16.5 \%)$, Out of 115 patients, $58(50.4 \%)$ patients had imaging findings suggestive of a GI primary. The tumour markers were checked in a total of $96(83.5 \%)$ out of 115 patients. These included carcino-embryonic antigen (CEA), alpha fetoprotein (AFP), carbohydrate antigen (CA-19.9) and cancer antigen (CA 125). The CEA levels were checked in 90/ $115(78.2 \%)$, and found to be raised in 62 (53.9), AFP was checked in a total of $46(40 \%)$, and was found to be elevated in 15 (13\%) Similarly, CA 19.9 was checked in $69(60 \%)$ of the total patients and was found to be high in $38(33 \%)$, The most common histological diagnoses with which patients were referred for endoscopy included adenocarcinoma $81(70.4 \%)$, followed by poorly differentiated metastatic carcinoma $16(13.9 \%)$, malignant fluid (ascites, pleural fluid) cytology in 9 (7.8\%), neuroendocrine tumor $3(2.6 \%)$, miscellaneous $4(3.4 \%)$ and squamous cell carcinoma $2(1.7 \%)$, as shown in Table-II.

These diagnoses were obtained by various means, as elaborated in Figure-1. Immunohistochemical stains, including cytokeratin (CK) 7 and CK 20, were checked in 21 patients. Of these, $10(47.6 \%)$ patients were positive for both CK 7 and CK 20, 8 (38.1\%) were positive for CK 20 only and $3(14.3 \%)$ were negative for both CK7 and CK 20.

Of these 115 patients, $75(66 \%)$ patients had a normal OGD, 17 (14.8\%) had gastropathy, 8 (6.9\%) had

Table-I: Baseline clinical characteristics.

\begin{tabular}{l|c}
\hline Variables & $\mathbf{n}(\mathbf{0})$ \\
\hline Mean \pm SD & $63 \pm 12.6$ years \\
Range & $22-88$ years \\
\hline Gender & $70(61 \%)$ \\
\hline Male & $45(39 \%)$ \\
Female & $87(75.6 \%)$ \\
\hline GI symptoms present & $61(53 \%)$ \\
Abdominal pain & $17(14.7 \%)$ \\
Weight loss & $5(4.3 \%)$ \\
Altered bowel habit & $2(1.8 \%)$ \\
Dysphagia & $1(0.9 \%)$ \\
Anorexia & $1(0.9 \%)$ \\
Vomiting & $28(24.4 \%)$ \\
Without GI symptoms &
\end{tabular}


changes of portal hypertension and 7 (6.1\%), patients had evidence of extrinsic compression. Similarly, 84 out of $115(73 \%)$ patients had a normal colonoscopy, $13(11.3 \%)$ had hemorrhoids only, whereas $8(7 \%)$ had polyps, histopathology of which showed no evidence of malignancy, 5 (4.3\%) of these patients had external colonic compression as shown in Table-III. In none of the patients included in the study, a definitive diagnosis of primary GI tumour obtained from OGD and/or colonoscopy.

Table-II: Details of various investigative modalities used.

\begin{tabular}{|c|c|}
\hline $\begin{array}{l}\text { Investigative Modalities } \\
\text { Suggesting GI Primary }\end{array}$ & n $(\%)$ \\
\hline \multicolumn{2}{|l|}{ Imaging Findings } \\
\hline $\begin{array}{l}\text { Metastases } \\
\text { Liver } \\
\text { Multiple sites } \\
\text { Pulmonary } \\
\text { Brain } \\
\text { Bone } \\
\text { Lymphadenopathy } \\
\text { Peritoneal thickening } \\
\text { Ascites } \\
\text { Gastric wall thickening }\end{array}$ & $\begin{array}{c}66(57.4 \%) \\
40(34.8) \\
19(16.5) \\
5(4.3) \\
2(0.9) \\
2(0.9) \\
10(7) \\
15(13) \\
7(6.1) \\
3(2.6) \\
\end{array}$ \\
\hline \multicolumn{2}{|l|}{ Tumour Markers } \\
\hline $\begin{array}{l}\text { Carcinoembryonic antigen } \\
\text { Median (IQR) } \\
\text { Alpha fetoprotein } \\
\text { Median (IQR) } \\
\text { Carbohydrate antigen-19.9 } \\
\text { Median (IQR) }\end{array}$ & $\begin{array}{l}2.69(1-1761) \\
86(2-100,000)\end{array}$ \\
\hline \multicolumn{2}{|l|}{ Histopathological Diagnoses } \\
\hline $\begin{array}{l}\text { Adenocarcinoma } \\
\text { Poorly diff metastatic } \\
\text { carcinoma } \\
\text { Neuroendocrine tumour } \\
\text { Squamous cell carcinoma } \\
\text { Miscellaneous } \\
\text { Papillary carcinoma } \\
\text { Epitheloid tumour } \\
\text { Malignant cytology }\end{array}$ & $\begin{array}{c}81(70.4) \\
16(13.9) \\
3(2.6) \\
2(1.7) \\
4(3.4) \\
2(1.7) \\
2(1.8) \\
9(7.8)\end{array}$ \\
\hline \multicolumn{2}{|c|}{ Table-III: Endoscopic findings. } \\
\hline Endoscopic findings & n (\%) \\
\hline \multicolumn{2}{|c|}{ Oesophago-Gastroduodenoscopy (OGD) } \\
\hline $\begin{array}{l}\text { Normal } \\
\text { Gastrpathy } \\
\text { Portal hypertension } \\
\text { Extrinsic compression }\end{array}$ & $\begin{array}{c}75(66) \\
12(14.8) \\
8(6.9) \\
7(6.1)\end{array}$ \\
\hline \multicolumn{2}{|l|}{ Colonoscopy } \\
\hline $\begin{array}{l}\text { Normal } \\
\text { Hemorrhoids } \\
\text { Polyps } \\
\text { Extrinsic compression }\end{array}$ & $\begin{array}{c}84(73) \\
13(11.3) \\
8(7) \\
5(4.3)\end{array}$ \\
\hline
\end{tabular}

\section{DISCUSSION}

According to the European Society for Medical Oncology (ESMO), the National Comprehensive
Cancer Network (NCCN) and the National Institute for Health and Care Excellence (NICE) guidelines on the diagnosis of CUP, OGD and colonoscopy should be performed only in patients who either have gastrointestinal (GI) symptoms or in those with imaging characteristics or immunohistochemical stains favoring the gastrointestinal tract as a source of primary tumour. ${ }^{10-12}$

In our study, we found that CUP is a cancer of elderly patients, most commonly occurring in the $6^{\text {th }}$ decade of life and is also more common in men $(60.9 \%$ vs. $39.9 \%$ ) than in women, as opposed to the figures stated in a population-based study done in Sweden, ${ }^{13}$ who reported a male to female proportion of $44.8 \mathrm{vs}$. $55.2 \%$ respectively. In another study by Saliminejad et al, ${ }^{14}$ The male to female proportion was equal. The mean age of presentation in our cohort was 63.3 years. In comparison, Abbruzzese et al, ${ }^{15}$ reported a median age of 59 years, while Levi et al, ${ }^{16}$ reported a median age of 70 years.

Most of our patients had abdominal pain $(53 \%, \mathrm{n}$ : $61)$ as a presenting complaint, while $24.3 \%(n=28)$ had no GI symptoms. The proportion of abdominal pain in our study group was similar to a study conducted by Usmani et $a l^{2}(50 \%)$ however, they reported a higher proportion of non-GI symptoms (45\%).

The most common tissue diagnoses of the metastatic site seen in our cohort was adenocarcinoma in $70.4 \%$ of patients, while $13.9 \%$ had poorly differentiated metastatic carcinoma, followed by malignant cytology in $7.8 \%$, neuroendocrine tumour in $3(2.6 \%)$, and squamous cell carcinoma in $2(1.7 \%)$. The commonest of the two tissue types are comparable to the study done by Abbruzzese et al, ${ }^{15}$ with their figures of adenocarcinoma and metastatic carcinoma of 58.1 and $29.4 \%$ respectively. However, the other study from our own country, by Usmani et al, ${ }^{2}$ revealed somewhat different results, apart from adenocarcinoma being the most common tissue type (75.5\%). Their second most common tissue type was neuroendocrine tumour, being a mere $3.4 \%$ of the total cohort $(n=86)$. In one study, by Petrović et al, ${ }^{17}$ histopathology review of biopsy specimens revealed adenocarcinoma to be $70 \%$, poorly differentiated carcinoma $20 \%$, squamous cell carcinoma $10 \%$, and poorly differentiated neoplasm $5 \%$.

Looking at the primary end point, our study did not demonstrate a single case of a primary tumour being detected by OGD and colonoscopy. In comparison, Usmani et al, ${ }^{2}$ reported a primary cancer detection rate of $7 \%$, or $3.5 \%$ each with OGD and colonoscopy. 
Saliminejad et al, ${ }^{14}$ analyzed the yield and cost of colonoscopy in patients with metastatic cancer of unknown primary. They reported primary cancer detection rates of only $1 \%$ and concluded that colonoscopy is not costeffective in seeking a primary in CUP. Overall, the data on primary tumour detection is quite scarce and no other studies could be found to support these figures. Additionally, the guidelines also advocate using OGD and colonoscopy based on symptoms or imaging findings. Nevertheless, these recommendations are largely anecdotal and require further studies to confirm this strategy. ${ }^{10-12}$

Bayrak et al, ${ }^{18}$ reported an immunohistochemical stain pattern of CK 7/20, showing CK7-/CK20+ being found in $64 \%$ of colorectal cancers and $5 \%$ of gastric cancers. Similarly CK7+/CK20+ was seen in $20 \%$ of colorectal and $48 \%$ of gastric cancers, while a CK7+/ CK20- pattern was seen in $2 \%$ of colorectal cancers. Keeping this in view, our study does raise some important questions. Imaging characteristics, raised tumour markers and immunohistochemical stains are all considered to be good predictors of a possible primary tumour in the GI tract. In our study, 62/115 (53.9\%) of patients had raised CEA levels, 58/115 (50.4\%) of our patients had imaging characteristics suggestive of a GI primary, and positive immune stains (both CK7 and CK20) were seen in 10/115 (8.6\%).

\section{LIMITATION OF STUDY}

In this relatively small and specific study population sample in a cancer specialized center, where patients are referred from peripheries after extensive work up, we were not able to find a GI primary in any of these patients, suggesting that the value of these investigations when referring for GI work up may need further discussion and review.

\section{CONCLUSION}

Our results concluded that OGD and colonoscopy, when done collectively as diagnostic procedures to look for a primary tumour, have no role in the evaluation of patients with CUP. Our results are largely in concordance with similar studies done on the subject under discussion and in light of these results, it is recommended that larger prospective studies be carried out, to help decide whether the guidelines for the use of OGD and colonoscopy in detecting CUP should be revised.

\section{Conflict of Interest: None.}

\section{Authors' Contribution}

MI: Conception, design, data analysis \& intellectual collection, RSAK: Conception, design, data analysis \& intellectual collection, FS: Conception, design, data analysis
\& intellectual collection, MAY: Conception, design, data analysis \& intellectual collection.

\section{REFERENCES}

1. Geropoulos G, Mitsos S, Lampridis S, Hayward M, Scarci M, Panagiotopoulos N. Carcinoma of unknown primary abuts left clavicle: Case report and review of the literature. Int J Surg Case Rep 2020; 67(1): 106-119.

2. Usmani MT, Khalid AB, Shah SH, Ahmad T, Hamid SS, Jafri SM. Yield of esophagogastro duodenoscopy and colonoscopy in cancer of unknown primary. Pak J Med Sci 2013; 29(2): 523-528.

3. Fizazi K, Greco FA, Pavlidis N, Pentheroudakis G, ESMO guide-lines working group. cancers of unknown primary site: ESMO clinical practice guidelines for diagnosis, treatment and follow-up. Ann Oncol 2011; 22(Suppl-6): vi64-68.

4. Pavlidis N, Briasoulis E, Hainsworth J, Greco FA. Diagnostic and therapeutic management of cancer of an unknown primary. Eur J Canc 2003; 39(14): 1990-2005.

5. Oien KA, Dennis JL. Diagnostic work-up of carcinoma of unknown primary: from immunohistochemistry to molecular profiling. Ann Oncol 2012; 23(suppl-10): x271-277.

6. Pavlidis N, Khaled H, Gaafar R. A mini review on cancer of unknown primary site: a clinical puzzle for the oncologists. J Adv Res 2015; 6(3): 375-382.

7. Adams HJ, de Klerk JM, Heggelman BG, Dubois SV, Kwee TC. Malignancy rate of biopsied suspicious bone lesions identified on FDG PET/CT. Eur J Nucl Med Mol Imag 2016; 43(7): 1231-1238.

8. Vikeså J, Møller AK, Kaczkowski B, Borup R, Winther O, Henao R, et al. Cancers of unknown primary origin (CUP) are characte-rized by chromosomal instability (CIN) compared to metastasis of know origin. BMC Canc 2015; 15(1): 151-155.

9. Tomuleasa C, Zaharie F, Muresan MS, Pop L, Fekete Z, Dima D, et al. How to diagnose and treat a cancer of unknown primary site. J Gastrointestin Liver Dis 2017; 26(2): 69-79.

10. Fizazi K, Greco FA, Pavlidis N, Daugaard G, Oien K, Pentheroudakis G. Cancers of unknown primary site: ESMO clinical practice guidelines for diagnosis, treatment and follow-up. Ann Oncol 2015; 26(suppl-5): v133-138.

11. National Institute for Clinical Excellence. Metastatic malignant disease of unknown primary origin in adults: diagnosis and management. NICE guideline (CG104). 2010, [Internet] Available at: https://www.nice.org.uk/guidance/cg104 (Accessed on December 6, 2019)

12. Ettinger DS, Handorf CR, Agulnik M, Bowles DW, Cates JM, Cristea $\mathrm{M}$, et al. Occult primary, version 3.2014. J Natl Compr Canc Netw 2014; 12(7): 969-974.

13. Shu $X$, Sundquist $K$, Sundquist J, Hemminki K. Time trends in incidence, causes of death, and survival of cancer of unknown primary in Sweden. Eur J Canc Prev 2012; 21(3): 281-288.

14. Saliminejad M, Bemanian S, Ho A, Spiegel B, Laine L. The yield and cost of colonoscopy in patients with metastatic cancer of unknown primary. Aliment Pharmacol Ther 2013; 38(6): 628-633.

15. Abbruzzese JL, Abbruzzese MC, Hess KR, Raber MN, Lenzi R. Unknown primary carcinoma: natural history and prognostic factors in 657 consecutive patients. J Clin Oncol 1994; 12(6): 1272-1280.

16. Levi F, Te VC, Erler G, Randimbison L, La Vecchia C. Epidemiology of unknown primary tumours. Eur J Canc 2002; 38(13): 1810-1812.

17. Petrović D, Muzikravić L, Jovanović D. Metastases of unknown origin: Principles of diagnosis and treatment. Medicinski Pregl 2007; 60(1-2): 29-36.

18. Bayrak R, Haltas $H$, Yenidunya $S$. The value of CDX2 and cytokeratins 7 and 20 expression in differentiating colorectal adenocarcinomas from extraintestinal gastrointestinal adenocarcino-mas: cytokeratin 7-/20+ phenotype is more specific than CDX2 antibody. Diagn Pathol 2012; 7(1): 9-12. 